\title{
Dispersion trimming for mid-infrared supercontinuum generation in a hybrid chalcogenide/silicon-germanium waveguide
}

\author{
Milan Sinobad, ${ }^{1,2}$ (D) Alberto Della Torre, ${ }^{1, *}$ Barry Luther-Davis, ${ }^{3}$ Pan Ma, $^{3}$ Stephen Madden, ${ }^{3}$ \\ Sukanta Debbarma, ${ }^{3}$ Khu Vu, ${ }^{3}$ David J. Moss, ${ }^{4}$ Arnan Mitchell, ${ }^{2}$ Jean-Michel Hartmann, ${ }^{5}$ \\ Jean-Marc Fedeli, ${ }^{5}$ Christelle Monat, ${ }^{1}$ and Christian Grillet ${ }^{1}$ \\ ${ }^{1}$ Université de Lyon, Institut des Nanotechnologies de Lyon (INL), 69131 Ecully, France \\ ${ }^{2}$ School of Engineering, RMIT University, Melbourne, VIC 3001, Australia \\ ${ }^{3}$ Laser Physics Center, Australian National University, Canberra, ACT 0100, Australia \\ ${ }^{4}$ Centre for Microphotonics, Swinburne University of Technology, Hawthorn, VIC 3122, Australia \\ ${ }^{5}$ Université Grenoble Alpes, CEA-Leti, 38054 Grenoble Cedex 9, France \\ ${ }^{*}$ Corresponding author: alberto.della-torre@ec-lyon.fr
}

Received 15 October 2018; revised 2 December 2018; accepted 3 December 2018; posted 5 December 2018 (Doc. ID 348366); published 8 January 2019

\begin{abstract}
We report a simple post-process technique that harnesses a hybrid chalcogenide/silicon-germanium system for the control of waveguide dispersion. By adding a chalcogenide top cladding to a $\mathrm{SiGe/Si}$ waveguide, we can substantially change the dispersive properties, which underpin the generation of a supercontinuum. In our particular example, we experimentally show that a shift from anomalous to normal dispersion takes place. We numerically study the dispersion dependence on the chalcogenide thickness and show how to use this additional degree of freedom to control the position of the zero dispersion wavelengths and hence the spectral span of the supercontinuum. Finally, we compare our approach with more traditional techniques that use geometry for dispersion tailoring. () 2019 Optical Society of America
\end{abstract}

https://doi.org/10.1364/JOSAB.36.000A98

\section{INTRODUCTION}

Broadband supercontinuum sources can be used advantageously in a wide variety of fields, from high-bit-rate communications and wavelength multiplexing [1] to biophotonics [2]. The mid-infrared (mid-IR) spectral region (covering the 3-20 $\mu \mathrm{m}$ wavelength range) is of great interest because of the large number of potential applications from biomedical imaging and medical and environmental sensors to food quality analysis and security [3-9]. Indeed, many chemical compounds have strong spectral fingerprints in this region due to their resonant frequencies associated with the vibrational and rotational modes of molecules. In this context, on-chip broadband midIR light sources, like supercontinua, are key for the development of efficient compact mid-IR spectroscopic platforms.

Mid-IR supercontinuum generation has already been demonstrated using different integrated platforms, e.g., chalcogenide [10], silicon-on-insulator waveguides [11,12], and silicon-on-sapphire waveguides $[13,14]$. Recently, the (theoretically foreseen) strong nonlinear properties [15], wide transparency window from 3 to $15 \mu \mathrm{m}$, and CMOS compatibility of germanium $[6,16,17]$ have generated increasing interest in germanium-based platforms. Generally, SiGe alloys are used to combine the superior nonlinear properties of germanium with an additional design parameter (the germanium content of the alloy) for a better control of the nonlinear properties of the waveguide (nonlinear index, multiphoton absorption, and dispersion) and to limit the waveguide/substrate lattice mismatch. The first demonstration of nonlinear effects in a SiGe waveguide was reported by Hammani et al. in 2014, showing mid-to-near-infrared conversion by four-wave mixing in a graded-index $\mathrm{SiGe}$ on a silicon waveguide [18], followed by the demonstration of supercontinuum generation from 1.45 to $2.79 \mu \mathrm{m}$ on the same platform [19]. Low propagation losses have been reported in graded-index SiGe waveguides [20,21], and a complete characterization of the nonlinear optical response of step-index $\mathrm{Si}_{0.6} \mathrm{Ge}_{0.4}$ on $\mathrm{Si}$ waveguides was performed by Carletti et al. in 2015 [22,23]. The latter work eventually led to our recent demonstration of the first supercontinuum generated across more than one octave (from 3 up to $8.5 \mu \mathrm{m}$ ) in $\mathrm{SiGe}$ waveguides [24]. In that work, an air cladding was used to achieve low anomalous dispersion in spite of the low index contrast between the SiGe core and the Si substrate. Controlling the waveguide dispersion is key to governing the nonlinear dynamics and hence the properties 
(bandwidth, coherence etc.,) of the generated supercontinuum. Sophisticated waveguide geometries have been proposed to that end. For instance, Bao et al. numerically studied a double-slot waveguide to obtain a flat and low dispersion [25] and a notch waveguide enabled Nader $e t$ al. to tune the zero-dispersion wavelength (ZDW) [14]. In all these demonstrations, however, the dispersive properties are set at the design stage and cannot be adjusted once the device has been fabricated [11-14,24,25]. Hence, they are subject to fabrication inaccuracies, surface contamination, and the presence of defects. Post-process tuning mechanisms are therefore of great interest to adjust or correct a posteriori the waveguide dispersion to match the target value.

Here we show that it is possible to fine-tune the dispersion profile a posteriori by adding a chalcogenide cladding layer on top of a highly nonlinear $\mathrm{SiGe}$ waveguide, introducing a simple post-processing tool to control the supercontinuum dynamics and its properties.

Several approaches have been proposed for post-process tuning of optical properties in optical fibers $[26,27]$ and photonic crystals [28-31]. However, the restricted number of design parameters makes post-process dispersion control of waveguides trickier. In this regard, the addition of a chalcogenide top cladding appears as a simple and elegant tool for posttrimming the dispersion, depending on the actual structure produced by fabrication. Chalcogenide glasses have been used for post-tuning distributed feedback lasers [32], quantum cascade lasers [33], photonic crystal cavities [34-36], and photonic crystal waveguides [37]. Here we report a proof of concept demonstration in which the deposition of a chalcogenide top cladding on nonlinear $\mathrm{SiGe} / \mathrm{Si}$ waveguides enables us to tune the group velocity dispersion of the fundamental mode. In our example, the group velocity dispersion changes from anomalous to normal at the pump wavelength, enabling us to observe supercontinuum generation from 3.1 up to $5.5 \mu \mathrm{m}$ in the normal dispersion regime. This demonstrates that the heterogeneous integration of materials can be used as a post-processing technique to tune the waveguide dispersive properties and therefore control supercontinuum generation. We numerically show that the dispersion can be further adjusted by setting the thickness of the deposited chalcogenide layer within a reasonable $100 \mathrm{~nm}$ resolution tolerance. The related effect on dispersion is equivalent to enlarging the waveguide by about $15 \%$ but without degrading the waveguide nonlinear response.

\section{DESIGN AND FABRICATION}

A $3.75 \times 2.7 \mu \mathrm{m}^{2}$ cross section, $5 \mathrm{~cm}$ long $\mathrm{Si}_{0.6} \mathrm{Ge}_{0.4}$ on a $\mathrm{Si}$ waveguide (see [24] for details on the fabrication process) was coated with a $1.26 \mu \mathrm{m}$ thick layer of a chalcogenide $\mathrm{Ge}_{11.5} \mathrm{As}_{24} \mathrm{Se}_{64.5}$, deposited by thermal evaporation [38]. The chalcogenide refractive index is considered constant and equal to 2.6 [39]. The waveguide is operated as a transverse-electric (TE) single-mode structure at the pump wavelength of $4.15 \mu \mathrm{m}$. Figure 1(a) shows scanning electron microscope (SEM) and atomic force microscope (AFM) images (top and bottom, respectively) of the waveguide surrounded by the chalcogenide layer. The top parts of Figs. 1(b) and 1(c) show the schematic of the waveguide before and after the deposition of the chalcogenide, respectively, while the corresponding simulated mode profiles (log scale) at $4.15 \mu \mathrm{m}$ are shown in the bottom parts. Table 1 summarizes the key parameters of the waveguide, with and without the chalcogenide top cladding, simulated by a finite-difference mode solver. Due to the low refractive index difference between the core and top cladding, the effective area of the mode slightly increases by $\sim 10 \%$ when the chalcogenide cladding is added; however, the mode confinement in the core of the waveguide remains unaffected and high ( 94\%). In principle, this allows us to maintain a high nonlinear efficiency, as dictated by the strong light interaction with the nonlinear SiGe core material.

Even though the influence of the cladding on the linear optical properties of the waveguide was not experimentally investigated, the relatively low mode energy overlap with the chalcogenide top cladding $(0.52 \%$ at $4.15 \mu \mathrm{m}$, see Table 1$)$ suggests that the impact of the chalcogenide surface roughness [Fig. 1(a)] on optical losses is limited. Scattering losses are dominant in high-index contrast waveguides and have less impact in the mid-infrared as they scale as $\lambda^{-4}$ [7]. Indeed, numerical

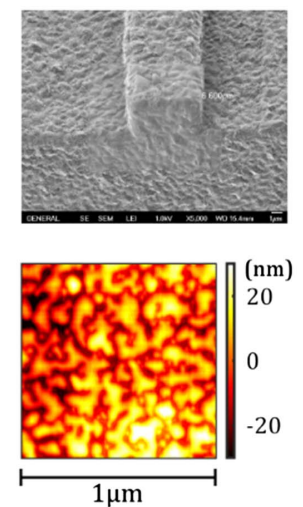

(a)

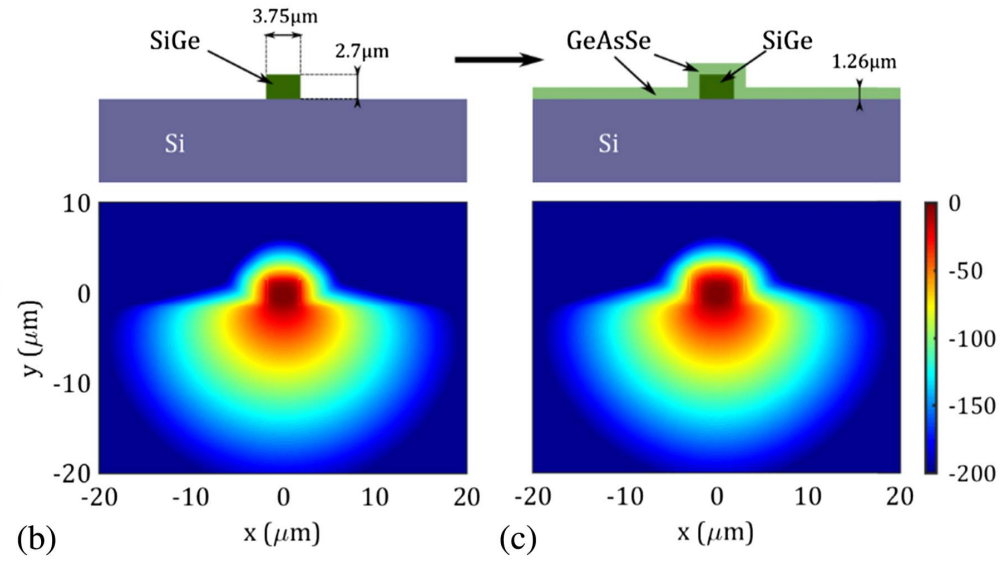

Fig. 1. (a) SEM (top) and AFM (bottom) images of the waveguide after the deposition of chalcogenide. Schematic (top) and simulated mode profile at $4.15 \mu \mathrm{m}$ for the waveguide before (b) the deposition of chalcogenide and for the (c) chalcogenide-clad waveguide. Simulations were performed using Lumerical and are represented with a logarithmic scale. 
Table 1. Cutoff Wavelength, Group Velocity Dispersion, Effective Area, Mode Energy Confinement, and Effective Index at the Pumping Wavelength $(4.15 \mu \mathrm{m})$ of the $3.75 \times$ $2.7 \mu^{2}$ Cross-Section Waveguide with an Air-Clad or a $1.26 \mu \mathrm{m}$ Thick Chalcogenide-Top Cladding

\begin{tabular}{lcc} 
Parameter & Air Clad & $\begin{array}{c}\text { Chalcogenide } \\
\text { Clad }\end{array}$ \\
\hline Cutoff wavelength $(\mu \mathrm{m})$ & 6 & 6.52 \\
$\mathrm{GVD}(\mathrm{ps} / \mathrm{nm} / \mathrm{km})$ at $4.15 \mu \mathrm{m}$ & 11.6 (anomalous) & -12.1 (normal) \\
$A_{\text {eff }}\left(\mu \mathrm{m}^{2}\right)$ at $4.15 \mu \mathrm{m}$ & 6.35 & 6.9 \\
$\begin{array}{l}\text { Mode confinement }(\%) \text { in the } \\
\text { SiGe core at } 4.15 \mu \mathrm{m}\end{array}$ & 94.3 & 94.4 \\
$\begin{array}{l}\text { Mode confinement }(\%) \text { in the } \\
\text { top cladding at } 4.15 \mu \mathrm{m}\end{array}$ & 0.0032 & 0.52 \\
Effective index at $4.15 \mu \mathrm{m}$ & 3.496 & 3.504 \\
\hline
\end{tabular}

simulations, taking into account the roughness measured by AFM $[\mathrm{rms}=15 \mathrm{~nm}$, see Fig. 1(a) bottom] at both the core/chalcogenide cladding and top chalcogenide interfaces, show that the impact of scattering is negligible $(\sim 0.2 \mathrm{~dB} / \mathrm{cm}$ of extra loss).

Figure 2(a) shows the spectral dependence of the effective area and the mode confinement factor in the core of the waveguide with and without the chalcogenide layer. The dispersion profile and the effective index are shown in Fig. 2(b). Considering the initial dimensions of the air-clad waveguide, the related anomalous dispersion at the pump wavelength shifts from anomalous to normal when the chalcogenide cladding is added. At the same time, the waveguide cutoff wavelength, which is characteristic of these vertically asymmetric waveguides [24], is slightly increased from 6 to $6.52 \mu \mathrm{m}$.

\section{SUPERCONTINUUM GENERATION}

We used the same setup, as described in Ref. [24], to couple light into the waveguide and collect the output spectra. The waveguide was pumped at $4.15 \mu \mathrm{m}$ with $\sim 200$ fs pulses delivered by a $200 \mathrm{~mW}$ tunable OPA laser source (MIROPA-fs, Hotlight Systems) with a repetition rate of $63 \mathrm{MHz}$. Optical waveplates and polarizers were used to control the power and polarization of the optical pulses, which were coupled to the waveguide with a set of chalcogenide lenses. The coupling loss was assumed to be the same at each facet and equal to those inferred in Ref. [24] $(-4.9 \mathrm{~dB})$. The output spectrum was recorded by a liquid-nitrogen-cooled MCT ( $\mathrm{HgCdTe})$ photodetector positioned at the output of the spectrometer. A lock-in detection technique was used to minimize the impact of thermal noise.

Figure 3 shows the experimental output spectra for increasing coupled peak power between 50 and $2.35 \mathrm{~kW}$ (corresponding to up to $50 \mathrm{~mW}$ of laser average power) obtained by pumping an air-clad waveguide [Fig. 3(a)] or a chalcogenide-clad waveguide [Fig. 3(b)], with the same SiGe core crosssection dimensions. The air-clad waveguide is slightly longer $(7 \mathrm{~cm})$ than the one with the chalcogenide cladding $(5 \mathrm{~cm})$. In both cases, a relatively broad supercontinuum was generated, spanning from 2.63 up to $6.18 \mu \mathrm{m}$ (with a $-30 \mathrm{~dB}$ bandwidth of $3.55 \mu \mathrm{m}$ ) and from 3.1 to $5.5 \mu \mathrm{m}$ (with a $-30 \mathrm{~dB}$ bandwidth of $2.4 \mu \mathrm{m}$ ) for the air cladding and chalcogenide cladding case, respectively. Some differences can be observed, which illustrate the changes to the dispersion caused by the addition of the chalcogenide top cladding. First, the spectrum is broader for the air-clad case as compared to the chalcogenide-clad case. This is expected for supercontinuum generated in the
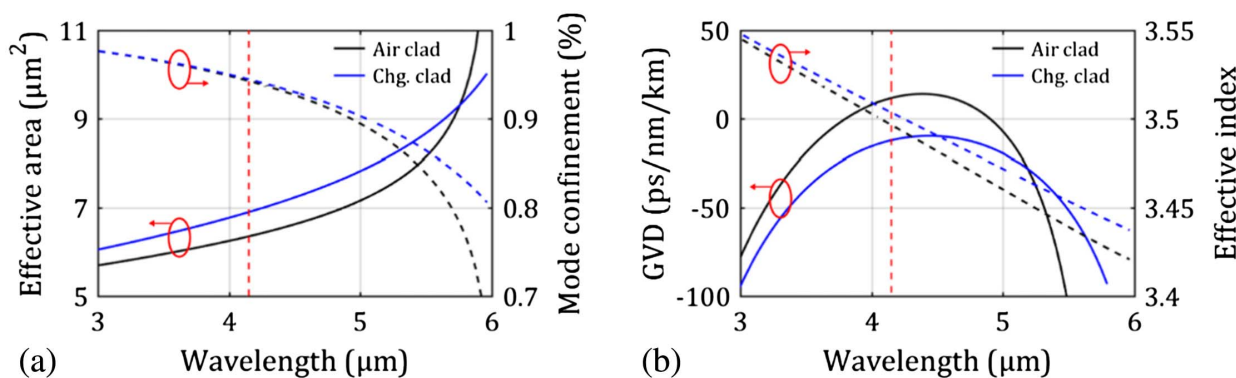

Fig. 2. (a) Calculated effective area (continuous lines) and mode confinement (dashed lines) for the air-clad (black) and chalcogenide-clad (blue) waveguide. (b) Calculated group velocity dispersion (continuous lines) and effective area of the mode (dashed lines) for the air-clad (black) and chalcogenide-clad (blue) waveguide. The red dashed line indicates the pump wavelength $(4.15 \mu \mathrm{m})$.
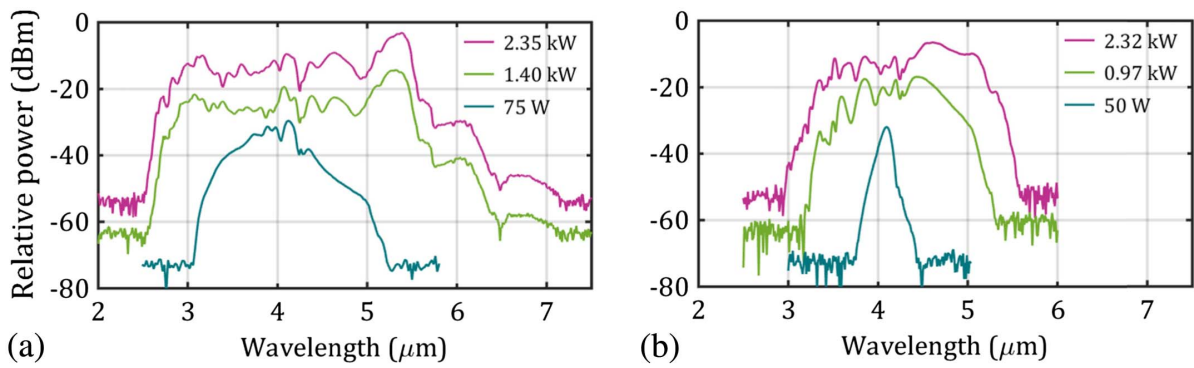

Fig. 3. Spectra measured out of the (a) $7 \mathrm{~cm}$ long air-cladded and (b) $5 \mathrm{~cm}$ long chalcogenide-cladded SiGe/Si waveguide with the same $3.75 \times$ $2.7 \mu \mathrm{m}^{2} \mathrm{SiGe}$ core cross-section dimensions for increasing coupled peak power. 

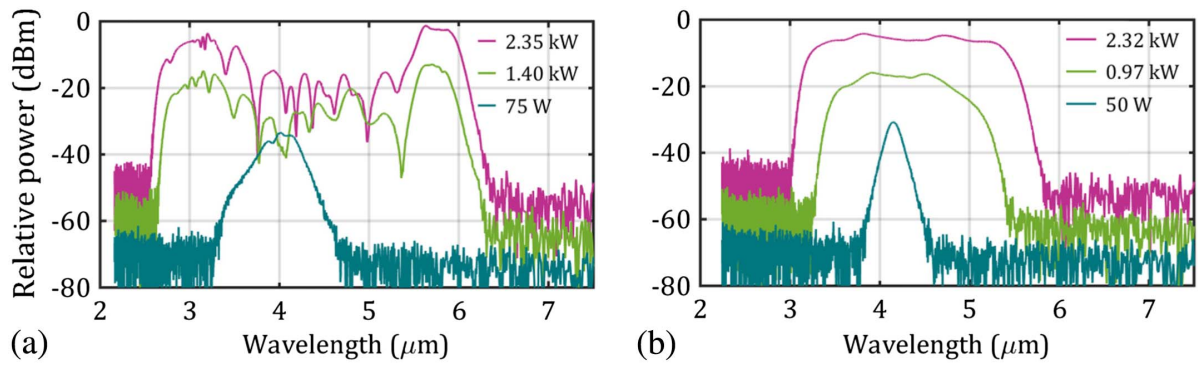

Fig. 4. Simulated spectra out of the (a) $7 \mathrm{~cm}$ long air-cladded and (b) $5 \mathrm{~cm}$ long chalcogenide-cladded waveguide for increasing coupled peak power.

anomalous dispersion rather than in the normal dispersion regime. Second, the supercontinuum amplitude is quite uneven in the long wavelength regime for the air-clad case, while the longer wavelength region of the supercontinuum spectrum generated by the chalcogenide top cladding device appears comparatively more regular.

This contrast in the long wavelength spectral signature is also a characteristic difference between the supercontinuum generation primarily in the anomalous dispersion regime (driven by soliton fission) and the normal dispersion regime (driven by self-phase modulation and optical wave breaking). We stress that the $2 \mathrm{~cm}$ difference in waveguide length should not be the cause of the observed differences between the supercontinua, as the spectrum of the air-clad anomalous dispersion case does not evolve much beyond the soliton fission length $(\sim 1.4 \mathrm{~cm})$, as was investigated in Ref. [24].

The supercontinuum generation process was simulated for both waveguides by numerically solving the nonlinear Schrödinger equation using the split-step Fourier method (see supplement in the Ref. [24] for more information). The Taylor expansion coefficients of the propagation constant were considered up to the tenth order. As a reasonable approximation, we used the same parameters for the linear loss, the nonlinear effects (Kerr and four photon absorption), the free-carrier absorption and dispersion, self-steepening, and the Raman effects as those used in Ref. [24] for the simulation of the air-clad waveguide pumped at $4.15 \mu \mathrm{m}$. Indeed, the chalcogenide compound is transparent deep into the mid-infrared, and its nonlinear contribution was neglected, as only $0.5 \%$ of the mode field energy overlaps with the chalcogenide cladding (see Table 1).
The experimental results of Fig. 3 are in good agreement with the simulations shown in Fig. 4; the bandwidth in particular is relatively well reproduced by the simulations. Some of the discrepancies, like the lobes at shorter wavelengths in the measured spectra on Fig. 3(b), are likely due to the coupling of light with higher-order modes and absorption to $\mathrm{CO} 2$ (around $4.2 \mu \mathrm{m}$ ) and hydrocarbons (around $3.2 \mu \mathrm{m}$ ) that are not taken into account in our simulation. These effects indeed equally affect the short wavelength spectral signature for both waveguides.

\section{RESULTS AND DISCUSSION}

\section{A. Dispersion Tailoring}

We have shown experimentally that by adding a chalcogenide layer on top of a $\mathrm{SiGe} / \mathrm{Si}$ waveguide, a change in the dispersion occurs, shifting, in this particular example, from an anomalous to normal dispersion at the pump wavelength. To expand on our findings, we performed a numerical analysis of the impact of the chalcogenide layer thickness on the group velocity dispersion [see Fig. 5(a)]. As the chalcogenide thickness increases, the overall dispersion gradually decreases, shifting towards normal values when depositing more than $500 \mathrm{~nm}$ of chalcogenide. This change is accompanied by a variation in the ZDWs and the flattening of the dispersion profile. The last trend is better observed in Fig. 5(b), which plots the corresponding third-order dispersion $\left(\beta_{3}\right)$. The third-order dispersion becomes smaller as the chalcogenide thickness increases, decreasing by more than a half (from 21.4 to $\left.9.8 \times 10^{-10} \mathrm{ps}^{3} / \mu \mathrm{m}\right)$ from the air-clad case to the $1.26 \mu \mathrm{m}$ thick chalcogenide-clad case, indicating that a flatter profile
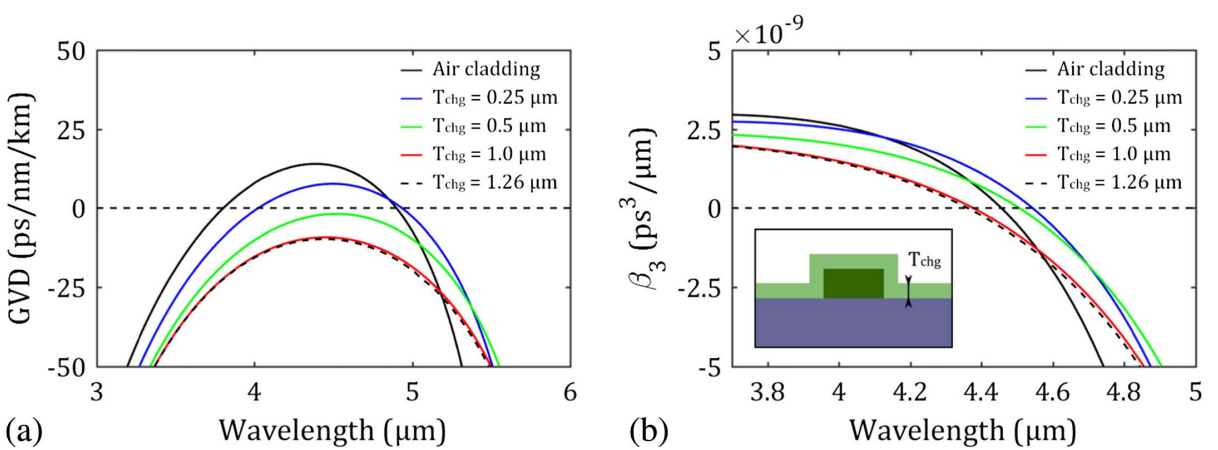

Fig. 5. (a) Calculated group velocity dispersion (GVD) for different thicknesses of the chalcogenide layer. (b) Corresponding third-order dispersion. The inset shows a schematic of the SiGe waveguide with chalcogenide cladding. 
of the group velocity dispersion is obtained. Moreover, the dispersion profile converges as the chalcogenide thickness approaches $1 \mu \mathrm{m}$, in agreement with the confinement of the mode in the waveguide core. As a flat profile of the dispersion is generally targeted for supercontinuum generation, both in the anomalous and in the normal dispersion regime, the possibility of controlling the dispersion profile and the position of the ZDWs by simply changing the thickness of the chalcogenide layer is a convenient post-process dispersion engineering tool. When supercontinuum generation in the anomalous dispersion regime is targeted, the position of the ZDWs determines the spectral location of the dispersive waves, which are located at the extreme parts of the spectrum. Therefore, the dispersive waves and thus the supercontinuum bandwidth and center wavelength can be selectively tuned by changing the thickness of the chalcogenide cladding.

The most common technique to tailor the dispersion profile of a waveguide with a given thickness is to change its width at the design stage. Figure 6 shows the group velocity dispersion of an air-clad 2.7 $\mu \mathrm{m}$ thick SiGe/Si waveguide for different widths. By increasing the width, we observe, similarly to the hybrid case, that the group velocity dispersion decreases, while the ZDWs are pushed toward longer wavelengths. However, the dispersion profile is less sensitive to the change of the waveguide width than to the thickness of the additional chalcogenide layer. For instance, a wide waveguide ( $25 \%$ larger $)$ is needed in order to substantially flatten the dispersion. Increasing the waveguide width could lead to a multi-mode regime and significantly increases the effective area, which may negatively impact the strength of nonlinearities, expressed through the factor $\gamma=2 \pi n_{2} / \lambda A_{\text {eff }}$, where $n_{2}$ is the nonlinear index and $A_{\text {eff }}$ is the effective area. By contrast, we have shown that the additional chalcogenide layer only moderately affects the effective area (see Table 1).

As a comparison example, the black dashed curve in Fig. 6 shows the dispersion profile of a $3.75 \mu \mathrm{m}$ wide waveguide with a $250 \mathrm{~nm}$ thick chalcogenide top cladding. The dispersion curve is similar to the one of a $4.3 \mu \mathrm{m}$ wide air-clad waveguide (green curve). The maximum group velocity dispersion shifts from $11.6 \mathrm{ps} / \mathrm{nm} / \mathrm{km}$ down to $7.53 \mathrm{ps} / \mathrm{nm} / \mathrm{km}$ by widening the air-clad waveguide and down to $7.67 \mathrm{ps} / \mathrm{nm} / \mathrm{km}$ by adding the chalcogenide layer, while the ZDWs shift from 3.8 and

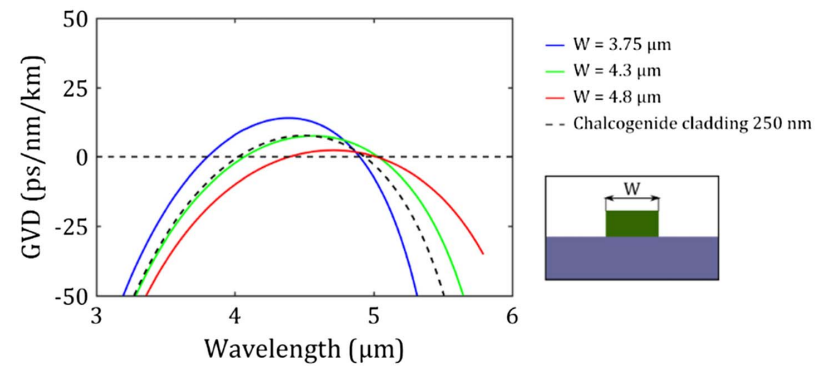

Fig. 6. Calculated group velocity dispersion for different waveguide widths (the thickness is fixed to $2.7 \mu \mathrm{m}$ ) and for a $3.75 \mu \mathrm{m}$ wide waveguide with $250 \mathrm{~nm}$ thick chalcogenide top cladding (dashed black curve). The dashed black line indicates the zero dispersion. The out inset shows a schematic of the air-clad waveguide.
$4.89 \mu \mathrm{m}$ to 4.07 and $5.03 \mu \mathrm{m}$ and to 4.02 and $4.94 \mu \mathrm{m}$, respectively.

While the tuning of the dispersion properties is very similar with the two techniques, the effective area of the mode is lower in the $250 \mathrm{~nm}$ thick chalcogenide-clad case $\left(6.77 \mu \mathrm{m}^{2}\right.$, compared to $7.14 \mu \mathrm{m}^{2}$ in the $4.3 \mu \mathrm{m}$ wide air-clad case), leading to a higher $\gamma$ factor. The undesirable increase in the effective area of the air-clad waveguide is even more severe when targeting normal dispersion for which a waveguide width of more than $5 \mu \mathrm{m}$ is needed, resulting in a $\gamma$ factor of less than $0.45(\mathrm{Wm})^{-1}$, compared to $\gamma=0.57(\mathrm{Wm})^{-1}$ for the chalcogenide-clad waveguide. Importantly, we reemphasize that the chalcogenide layer can be added as a post-process, and the thickness of the layer could be chosen to trim the dispersion properties after the fabricated waveguide is characterized. A thickness resolution of the chalcogenide layer around $100 \mathrm{~nm}$ is easily achievable.

\section{B. Outlook}

Besides the potential for adjusting the waveguide dispersion by controlling the thickness of a chalcogenide top cladding, the high photosensitivity of chalcogenide glasses [38] can be used to locally change their refractive index, density, and thickness through simply illuminating this material with light energy above their band gap. Exploiting the photosensitivity of these glasses is thus, in principle, an additional tool for posttrimming the properties of individual waveguides not only to relax the fabrication constraints but also to tailor the dispersion along the waveguide and to optimize the design of individual structures for different environments and applications [40]. Finally, although it was not exploited here, chalcogenide glasses possess a third-order optical nonlinearity up to a thousand times higher than silica glasses, [41] minimizing the potential negative impact that an additional top cladding might have on the effective nonlinear parameter.

\section{CONCLUSION}

We presented a simple post-process technique to fine-tune the dispersion profile of a nonlinear $\mathrm{SiGe}$ on an $\mathrm{Si}$ waveguide through adding a top chalcogenide layer for changing the properties of a chip-based generated supercontinuum. We demonstrated the utility of this approach through the generation of a mid-IR supercontinuum and have compared it with more traditional geometric changes of the waveguide dimensions.

Supercontinuum generation in both the normal and anomalous dispersion regimes requires group velocity dispersion profiles that are as low in magnitude and as flat as possible. Nanowaveguides that are used in integrated platforms can fulfill these strict requirements through design adjustment. They are, however, very sensitive to fabrication inaccuracies, surface roughness, surface contamination, and the presence of defects, resulting in deviations of the final device from the targeted dispersion profile. Therefore, a post-process approach that allows us to trim the group velocity dispersion depending on the actual structure produced by fabrication is a highly attractive complementary tool for optimizing chip-based supercontinuum generation. Finally, the wide transparency window of both the SiGe core and the chalcogenide cladding makes this 
platform well suited to extend mid-IR coherent supercontinuum generation toward longer wavelengths, covering a great part of the mid-IR region, with potential applications in biomedical imaging, medical and environmental sensors, food quality analysis, and security.

Funding. Agence Nationale de la Recherche (ANR) (ANR17-CE24-0028); H2020 European Research Council (ERC) (648546).

Acknowledgments. We acknowledge the support of the International Associated Laboratory between France and Australia (LIA ALPhFA), and author Christelle Monat acknowledges the support of the Institut Universitaire de France. We thank Aziz Benamrouche for the Atomic Force Microscope measurements of the chalcogenide surface.

\section{REFERENCES}

1. A. E. Willner, S. Khaleghi, M. R. Chitgarha, and O. F. Yilmaz, "Alloptical signal processing," J. Lightwave Technol. 32, 660-680 (2014).

2. H. Tu and S. A. Boppart, "Coherent fiber supercontinuum for biophotonics," Laser Photon. Rev. 7, 628-645 (2013).

3. L. Wang and B. Mizaikoff, "Application of multivariate data-analysis techniques to biomedical diagnostics based on mid-infrared spectroscopy," Anal. Bioanal. Chem. 391, 1641-1654 (2008).

4. B. Guo, Y. Wang, C. Peng, H. L. Zhang, G. P. Luo, and H. Q. Le, "Laser-based mid-infrared reflectance imaging of biological tissues," Opt. Express 12, 208-219 (2004).

5. M. Sieger and B. Mizaikoff, "Toward on-chip mid-infrared sensors," Anal. Chem. 88, 5562-5573 (2016).

6. R. Soref, "Mid-infrared photonics in silicon and germanium," Nat. Photonics 4, 495-497 (2010).

7. L. Zhang, A. M. Agarwal, L. C. Kimerling, and J. Michel, "Nonlinear group IV photonics based on silicon and germanium: from nearinfrared to mid-infrared," Nanophotonics 3, 247-268 (2014).

8. H. Lin, Z. Luo, T. Gu, L. C. Kimerling, K. Wada, A. Agarwal, and J. Hu, "Mid-infrared integrated photonics on silicon: a perspective," Nanophotonics 7, 393-420 (2017).

9. T. Hu, B. Dong, X. Luo, T. Liow, J. Song, C. Lee, and G. Lo, "Silicon photonic platforms for mid-infrared applications," Photon. Res. 5, 417-430 (2017).

10. Y. Yu, X. Gai, P. Ma, K. Vu, Z. Yang, R. Wang, D. Choi, S. Madden, and B. Luther-Davies, "Experimental demonstration of linearly polarized 2-10 $\mu \mathrm{m}$ supercontinuum generation in a chalcogenide rib waveguide," Opt. Lett. 41, 958-961 (2016).

11. R. K. W. Lau, M. R. E. Lamont, A. G. Griffith, Y. Okawachi, M. Lipson, and A. L. Gaeta, "Octave-spanning mid-infrared supercontinuum generation in silicon nanowaveguides," Opt. Lett. 39, 4518-4521 (2014).

12. R. Kou, T. Hatakeyama, J. Horng, J. Kang, Y. Wang, X. Zhang, and F. Wang, "Mid-IR broadband supercontinuum generation from a suspended silicon waveguide," Opt. Lett. 43, 1387-1390 (2018).

13. N. Singh, D. Hudson, Y. Yu, C. Grillet, S. D. Jackson, A. CasasBedoya, A. Read, P. Atanackovic, S. G. Duvall, S. Palomba, B. Luther-Davies, S. Madden, D. J. Moss, and B. J. Eggleton, "Midinfrared supercontinuum generation from 2 to $6 \mu \mathrm{m}$ in a silicon nanowire," Optica 2, 797-802 (2015).

14. N. Nader, D. L. Maser, F. C. Cruz, A. Kowligy, H. Timmers, J. Chiles, C. Fredrick, D. A. Westly, S. Woo Nam, R. P. Mirin, J. M. Shainline, and S. Diddams, "Versatile silicon-waveguide supercontinuum for coherent mid-infrared spectroscopy," APL Photon. 3, 036102 (2018).

15. N. K. Hon, R. Soref, and B. Jalali, "The third-order nonlinear optical coefficients of $\mathrm{Si}, \mathrm{Ge}$, and $\mathrm{Si}_{1-\mathrm{x}} \mathrm{Ge}_{\mathrm{x}}$ in the midwave and longwave infrared," J. Appl. Phys. 110, 011301 (2011).

16. G. Z. Mashanovich, C. J. Mitchell, J. Soler Penades, A. Z. Khokhar, C. G. Littlejohns, W. Cao, Z. Qu, S. Stankovic, F. Y. Gardes, T. Ben Masaud, H. M. H. Chong, V. Mittal, G. Senthil Murugan,
J. S. Wilkinson, A. C. Peacock, and M. Nedeljkovic, "Germanium midinfrared photonic devices," J. Lightwave Technol. 35, 624-630 (2017).

17. M. Nedeljkovic, J. Soler Penades, V. Mittal, G. Senthil Murugan, A. Z. Khokhar, C. Littlejohns, L. G. Carpenter, C. B. E. Gawith, J. S. Wilkinson, and G. Z. Mashanovich, "Germanium-on-silicon waveguides operating at mid-infrared wavelengths up to $8.5 \mu \mathrm{m}$," Opt. Express 25, 27431-27441 (2017).

18. K. Hammani, M. A. Ettabib, A. Bogris, A. Kapsalis, D. Syvridis, M. Brun, P. Labeye, S. Nicoletti, and P. Petropoulos, "Towards nonlinear conversion from mid-to near-infrared wavelengths using Silicon Germanium waveguide," Opt. Express 22, 9667-9674 (2014).

19. M. A. Ettabib, L. Xu, A. Bogris, A. Kapsalis, M. Belal, E. Lorent, P. Labeye, S. Nicoletti, K. Hammani, D. Syvridis, D. P. Shepherd, J. H. V. Price, D. J. Richardson, and P. Petropoulos, "Broadband telecom to mid-infrared supercontinuum generation in a dispersionengineered silicon germanium waveguide," Opt. Lett. 40, 41184121 (2015).

20. J. M. Ramirez, V. Vakarin, C. Gilles, J. Frigerio, A. Ballabio, P. Chaisakul, X. Le Roux, C. Alonso-Ramos, G. Maisons, L. Vivien, M. Carras, G. Isella, and D. Marris-Morini, "Low-loss Ge-rich $\mathrm{Si}_{0.2} \mathrm{Ge}_{0.8}$ waveguides for mid-infrared photonics," Opt. Lett. 42 , 105-108 (2017).

21. J. M. Ramirez, Q. Liu, V. Vakarin, J. Frigerio, A. Ballabio, X. Le Roux, D. Bouville, L. Vivien, G. Isella, and D. Marris-Morini, "Graded SiGe waveguides with broadband low-loss propagation in the mid infrared," Opt. Express 26, 870-877 (2018).

22. L. Carletti, P. Ma, Y. Yu, B. Luther-Davies, D. Hudson, C. Monat, R. Orobtchouk, S. Madden, D. J. Moss, M. Brun, S. Ortiz, P. Labeye, S. Nicoletti, and C. Grillet, "Nonlinear optical response of low loss silicon germanium waveguides in the mid-infrared," Opt. Express 23, 8261-8271 (2015).

23. L. Carletti, M. Sinobad, P. Ma, Y. Yu, D. Allioux, R. Orobtchouk, M. Brun, S. Ortiz, P. Labeye, J. M. Hartmann, S. Nicoletti, S. Madden, B. Luther-Davies, D. J. Moss, C. Monat, and C. Grillet, "Mid-infrared nonlinear optical response of $\mathrm{Si}-\mathrm{Ge}$ waveguides with ultra-short optical pulses," Opt. Express 23, 32202-32214 (2015).

24. M. Sinobad, C. Monat, B. Luther-Davies, P. Ma, S. Madden, D. J. Moss, A. Mitchell, D. Allioux, R. Orobtchouk, S. Boutami, J. M. Hartmann, J. M. Fedeli, and C. Grillet, "Mid-infrared octave spanning supercontinuum generation to $8.5 \mu \mathrm{m}$ in silicon-germanium waveguides," Optica 5, 360-366 (2018).

25. C. Bao, Y. Yan, L. Zhang, Y. Yue, N. Ahmed, A. M. Agarwal, L. C. Kimerling, J. Michel, and A. E. Willner, "Increased bandwidth with flattened and low dispersion in a horizontal double-slot silicon waveguide," J. Opt. Soc. Am. B 32, 26-30 (2015).

26. F. Lu and W. H. Knox, "Generation, characterization, and application of broadband coherent femtosecond visible pulses in dispersion micromanaged holey fibers," J. Opt. Soc. Am. B 23, 1221-1227 (2006).

27. G. Wong, L. Zang, M. S. Kang, and P. St. J. Russell, "Measurement of group-velocity dispersion of Bloch modes in photonic-crystal-fiber rocking filters," Opt. Lett. 35, 3982-3984 (2010).

28. J. Pan, Y. Huo, K. Yamanaka, S. Sandhu, L. Scaccabarozzi, R. Timp, M. L. Povinelli, S. Fan, M. M. Fejer, and J. S. Harris, "Aligning microcavity resonances in silicon photonic-crystal slabs using laserpumped thermal tuning," Appl. Phys. Lett. 92, 103114 (2008).

29. F. Raineri, C. Cojocaru, R. Raj, P. Monnier, and A. Levenson, "Tuning a two-dimensional photonic crystal resonance via optical carrier injection," Opt. Lett. 30, 64-66 (2005).

30. C. Grillet, C. Monat, C. L. Smith, M. W. Lee, S. Tomljenovic-Hanic, C. Karnutsch, and B. J. Eggleton, "Reconfigurable photonic crystal circuits," Laser Photon. Rev. 4, 192-204 (2010).

31. A. Casas Bedoya, P. Domachuk, C. Grillet, C. Monat, E. C. Mägi, E. $\mathrm{Li}$, and B. J. Eggleton, "Reconfigurable photonic crystal waveguides created by selective liquid infiltration," Opt. Express 20, 11046-11056 (2012).

32. T. K. Sudoh, Y. Nakano, and K. Tada, "Wavelength trimming technology for multiple-wavelength distributed-feedback laser arrays by photoinduced refractive index change," Electron. Lett. 33, 216-217 (1997).

33. S. Song, S. S. Howard, Z. Liu, A. O. Dirisu, C. F. Gmachl, and C. B. Arnold, "Mode tuning of quantum cascade lasers through optical 
processing of chalcogenide glass claddings," Appl. Phys. Lett. 89, 041115 (2006).

34. M. W. Lee, C. Grillet, S. Tomljenovic-Hanic, E. C. Mägi, D. J. Moss, B. J. Eggleton, X. Gai, S. Madden, D. Choi, D. A. P. Bulla, and B. Luther-Davies, "Photowritten high-Q cavities in two-dimensional chalcogenide glass photonic crystals," Opt. Lett. 34, 3671-3673 (2009).

35. A. Faraon, D. Englund, D. Bulla, B. Luther-Davies, B. J. Eggleton, N. Stoltz, P. Petroff, and J. Vučković, "Local tuning of photonic crystal cavities using chalcogenide glasses," Appl. Phys. Lett. 92, 043123 (2008).

36. M. W. Lee, C. Grillet, C. Monat, E. Mägi, S. Tomljenovic-Hanic, X. Gai, S. Madden, D. Choi, D. Bulla, B. Luther-Davies, and B. J. Eggleton, "Photosensitive and thermal nonlinear effects in chalcogenide photonic crystal cavities," Opt. Express 18, 26695-26703 (2010).

37. M. W. Lee, C. Grillet, C. L. C. Smith, D. J. Moss, B. J. Eggleton, D. Freeman, B. Luther-Davies, S. Madden, A. Rode, Y. Ruan, and
Y. Lee, "Photosensitive post tuning of chalcogenide photonic crystal waveguides," Opt. Express 15, 1277-1285 (2007).

38. D. A. P. Bulla, R. P. Wang, A. Prasad, A. V. Rode, S. J. Madden, and B. Luther-Davies, "On the properties and stability of thermally evaporated Ge-As-Se thin films," Appl. Phys. A 96, 615-625 (2009).

39. H. G. Dantanarayana, N. Abdel-Moneim, Z. Tang, L. Sojka, S. Sujecki, D. Furniss, A. B. Seddon, I. Kubat, O. Bang, and T. M. Benson, "Refractive index dispersion of chalcogenide glasses for ultra-high numerical-aperture fiber for mid-infrared supercontinuum generation," Opt. Mater. Express 4, 1444-1455 (2014).

40. Y. Ruan, M. Kim, Y. Lee, B. Luther-Davies, and A. Rode, "Fabrication of high-Q chalcogenide photonic crystal resonators by e-beam lithography," Appl. Phys. Lett. 90, 071102 (2007).

41. S. Dai, Y. Wang, X. Peng, P. Zhang, X. Wang, and Y. Xu, "A review of mid-infrared supercontinuum generation in chalcogenide glass fibers," Appl. Sci. 8, 707 (2018). 